\title{
Association of Craniovertebral Junction Anomalies, Klippel-Feil Syndrome, Ruptured Dermoid Cyst and Mirror Movement in One Patient: A Unique Case and Literature Review
}

\author{
Yu-kun ZHANG, Su-Min GENG, Pi-Nan LIU, Gang LV \\ Beijing Tiantan Hospital, Capital Medical University, Department of Neurosurgery, Beijing, China
}

\section{ABSTRACT}

The Klippel-Feil syndrome (KFS) has been reported to be associated with intracranial neoplasms, most frequently epidermoid or dermoid cysts. To our knowledge, however, patients who present with a posterior fossa dermoid cyst (DC) and KFS are extremely rare with only 24 previously reported cases in the English literature worldwide. Therefore, we present the first report of a patient with a craniocervical ruptured DC accompanied by craniovertebral junction (CVJ) anomalies, KFS and mirror movement. Meanwhile, a literature review of KFS accompanying with posterior fossa DC discusses these conditions from the embryological, anatomical, clinical and therapeutic perspectives. Additionally, the combination of CVJ anomalies, KFS and DC may represent a new syndrome that has previously gone unnoticed.

KEYWORDS: Craniovertebral junction, Dermoid cyst, Klippel-Feil syndrome, Mirror movement, Rupture

\section{INTRODUCTION}

Dermoid cysts (DCs) are rare neoplasms, accounting for $0.1-0.7 \%$ of all intracranial tumors $(20,29)$. In 1936 , Love and Kernohan first reported the association of DCs of the posterior cranial fossa with cervical fusion anomalies, particularly Klippel-Feil syndrome (KFS) (20). Subsequently, only 23 other cases of KFS-associated posterior cranial fossa DC (1, 3, 4, 7$10,13,15,19,20,23,25,27,28,34,35,40,41,44)$ have been reported in the English literature, as listed in Table I. Because these diseases are very rare, the association between DC and KFS may be pathophysiologically significant. We present the first report of a patient with a craniocervical ruptured DC accompanied by craniovertebral junction (CVJ) anomalies, KFS and mirror movement.

\section{CASE REPORT}

History. A 28-year-old unmarried woman presented with a five-month history of unsteady gait, which had worsened one month before admission. The onset of this symptom occurred without any obvious cause. She did not complain of headache, nausea or vomiting episodes. In addition, since birth, the patient had experienced involuntary movement of the contralateral hand while performing voluntary movements with the other hand, although this symptom had never previously caused distress. There was no family history of medical disorders.

Physical Examination. The patient, whose neck was short with a high neck/height ratio (1:20.5, normal 1:9) (32), had a low posterior hairline, extending to the level of the $\mathrm{C} 7$ spinous process (Figure 7A). Upon physical examination, the examiner was able to palpate one apparent pit in the 
nape of the patient's neck (Figure 7A), and flexion-extension motions were maintained to a limited extent (Figures 1B, C). Mirror movement was easily elicited and primarily confined to the hand. The patient had gait ataxia, as well as apparent clumsiness in performing the heel-knee-tibia test and finger-to-nose test; Romberg's sign was positive. The other neurological examinations were negative.
Radiographic Examination. Plain radiography and computed tomography (CT) of the head and neck revealed congenital fusions (classified as Type I of KFS) of C2-C7 and T1-T3 associated with platybasia or basilar invagination (Figures 1A$C, 2 A, B)(11)$. A three-dimensionally (3D) reconstructed CT of CVJ demonstrated spina bifida occulta from C2 to C7 (Figure 3A-C). Most notably, CT showed a low-density, calcified,
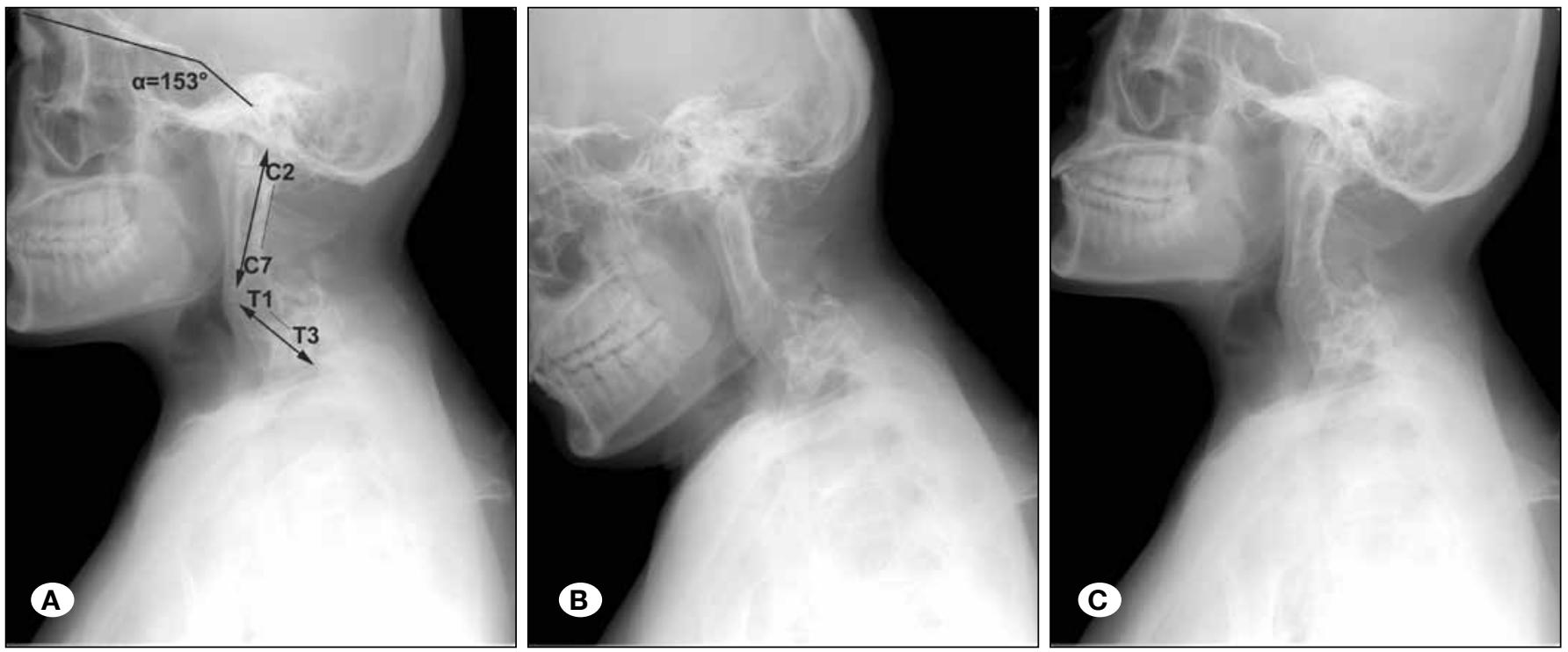

Figure 1: Plain cervical spine radiographs. A) Plain radiograph shows congenital fusions (Type I of KFS) (double arrowheads) with $153^{\circ}$ NTB angle of Welcker joining the nasion $(\mathrm{N})$, tuberculum $(\mathrm{T})$, and basion $(\mathrm{B})$. The normal angle should be less than $130^{\circ}(29)$, platybasia with an increased NTB angle. B, C) Flexion and extension of cervical spine.
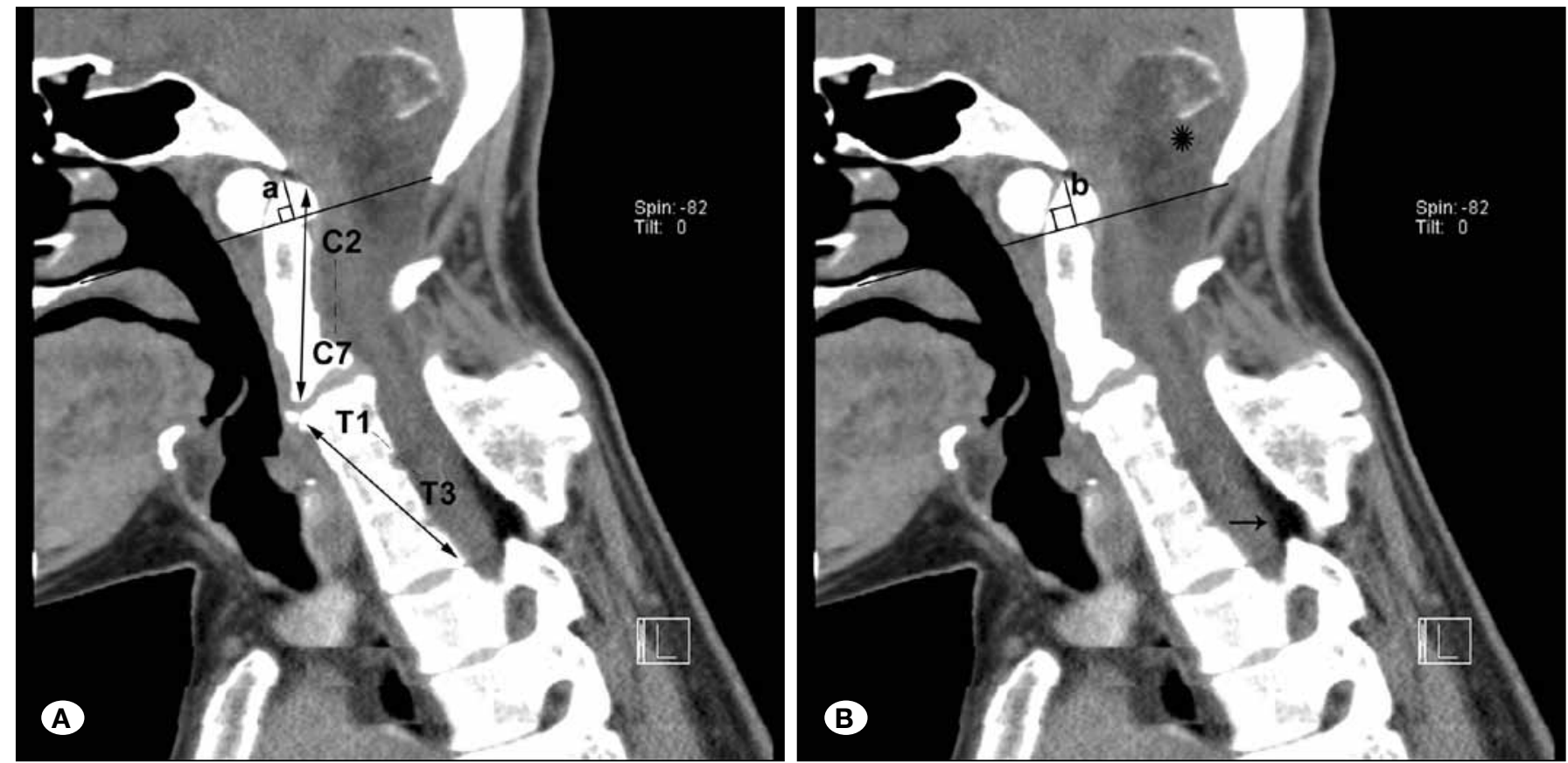

Figure 2: Midsagittal reconstructed images from cervical spine CT. A) Double arrowheads reveal congenital fusions (Type I of KFS) (11). Radiographic criteria for basilar invagination with dens protrudes $>2.5 \mathrm{~mm}(a=8.0 \mathrm{~mm})$ above Chamberlain's line between hard palate and opisthion (20). B) Another criteria with dens protrudes $>5.0 \mathrm{~mm}(b=9.8 \mathrm{~mm})$ above McGregor's line between hard palate and the lowest point of occiput (20). Besides, there are two lesions (asterisk and black arrow). 
circumscribed mass lesion (measured as $-70 \sim 170 \mathrm{Hu}$ ) around the foramen magnum. In addition, another lesion (measured as $-120 \mathrm{Hu}$ ) was found in the upper thoracic spinal canal. CT indicated that the second lesion was likely to be a lipoma (Figure 2A, B).

Magnetic resonance imaging (MRI) revealed one oval intradural mass located in the craniocervical area, measuring $28.6 \mathrm{~mm}$ anteroposteriorly and $50.3 \mathrm{~mm}$ rostrocaudally. The lesion was heterogeneous and had areas of hyper-, iso, and hypointensity on T1-weighted images and areas of hyperintensity on T2-weighted images (Figure 4A-C). In addition, multiple small, short T1WI and long T2WI signal lesions were scattered in the brain fissures, subarachnoid spaces and ventricles (Figure 4A, Figure 5A-D). These lesions were assumed to be fatty droplets derived from spontaneous rupture of the DC (Figure 5A).

3D CTA of the vertebral artery (VA) (Figure 6A-E) demonstrated that the third segment of the right vertebral artery (RVA) had an abnormal course, leaving the transverse foramen of the axis and passing into the foramen magnum below the occipitalized lateral mass of $\mathrm{C} 1$; this was classified as a Type I VA at the CVJ with occipitalization of the atlas (42). The second segment of the left vertebral artery (LVA) was obviously distorted from the osseous fusion of $\mathrm{C} 2-\mathrm{C} 7$. In addition, there was a fenestration of the vertebrobasilar artery.

Surgery. With the use of microsurgical instruments, total resection of the lesion was performed (Figure 7A-C). The anterior portion of the lesion was adherent to the dorsal surface of the brainstem, where an inconspicuous cleft was observed, as shown in the preoperative MRI (Figure 5A). Next, hydrocortisone was used to extensively irrigate the resection bed, in order to prevent postoperative chemical meningitis. No occipitocervical $(\mathrm{OC})$ fixation or fusion was performed during the surgery. After the successful operation, a postoperative neck collar was prescribed for one month.
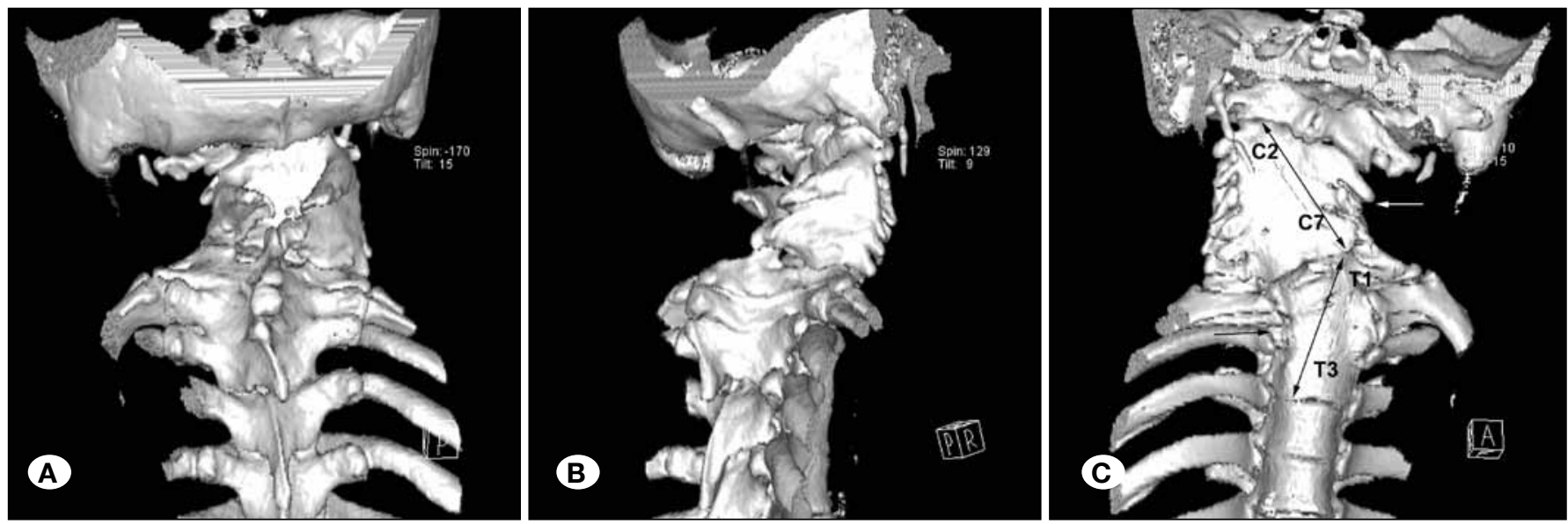

Figure 3: 3D CT reconstruction of craniovertebral region. A) Posterior view rendering of spina bifida occulta of C1-C7. B) Lateral view showing of defects of posterior arch of atlas. C. Anterior view indicating of scoliosis of congenital fusion (double arrowheads), bifid anterior arch of atlas, and atlantooccipital joint asymmetry, cervical and upper thoracic spine concave to right (white arrow) and left (black arrow) respectively.
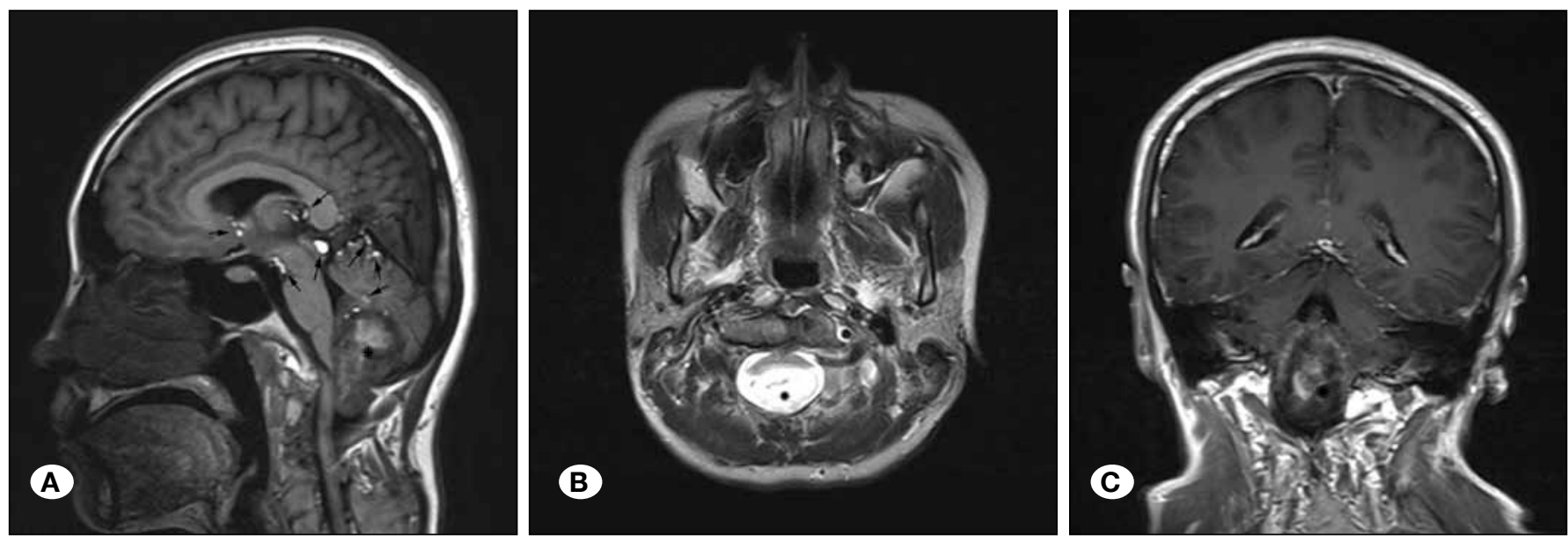

Figure 4: Preoperative MRI of head and neck. A) Sagittal T1WI reveals one heterogeneous mass (asterisk) and scattered droplets (arrows). B) Axial T2WI shows a hyper-intense mass (asterisk). C) Coronal contrast-enhanced T1WI of the lesion (asterisk). 
Pathological Findings. Microscopic examination of the excised mass revealed the presence of a classic DC with calcification (Figure 8).

Postoperative Course. The course was uneventful, and the patient was discharged with no further neurological deficits on the $10^{\text {th }}$ day after the surgery.
Follow-up. After six months of follow-up, the patient's preoperative symptoms had resolved, with the exception of mirror movement. Compared with the preoperative MRI findings (Figures 4A-C, 5A-D), postoperative MRI of the head showed no recurrence of the lesion; the fat droplets were stable without enlargement, reduction or apparent displacement (Figure 9).
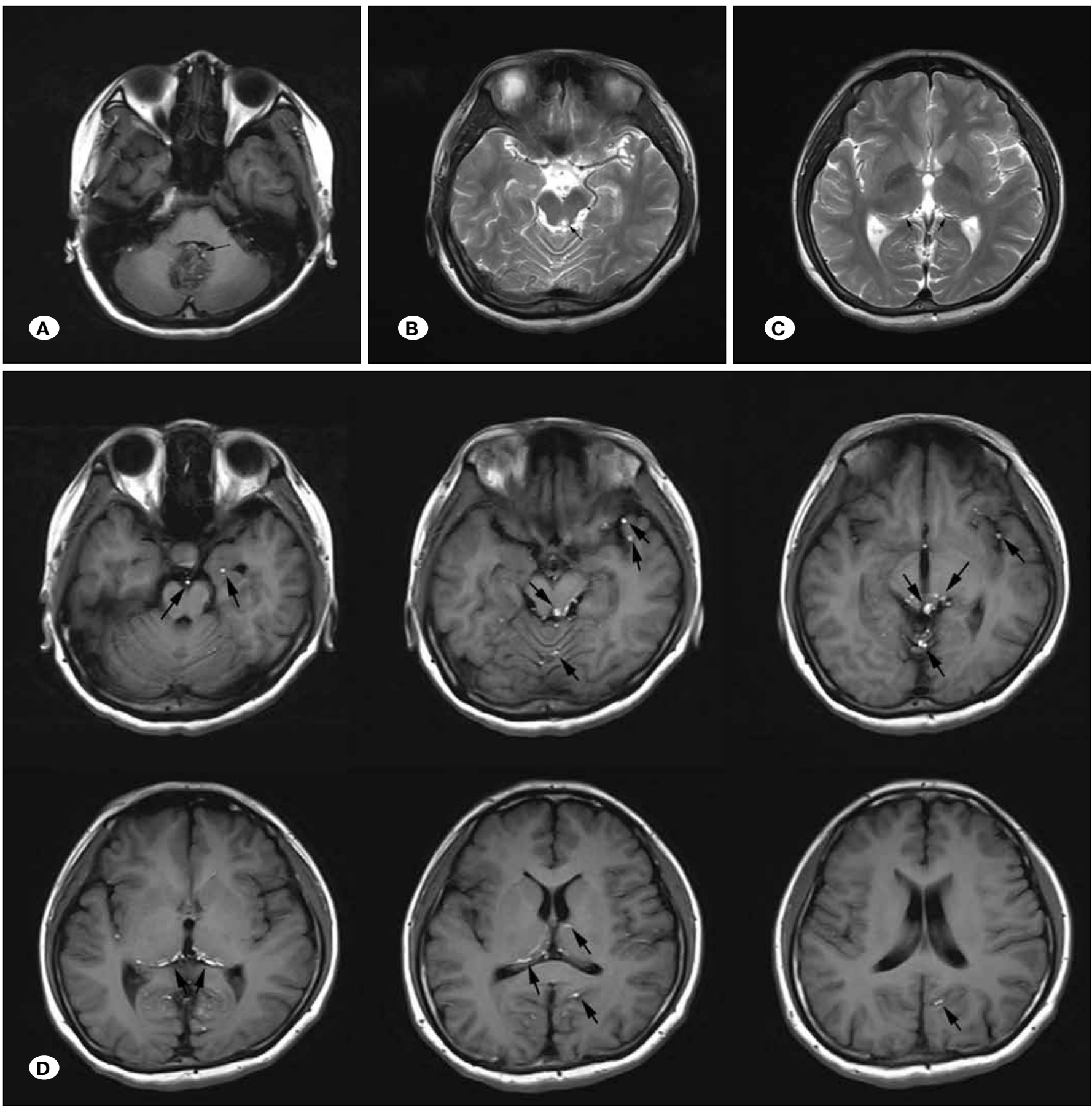

Figure 5: A series of MR images showing the scattered fat droplets. A) There is an inconspicuous cleft (arrow). B, C) They show the hyperintense fat droplets (arrows) on T2WI. D) It describes the hyperintense fat droplets ( arrows) on T1WI. 

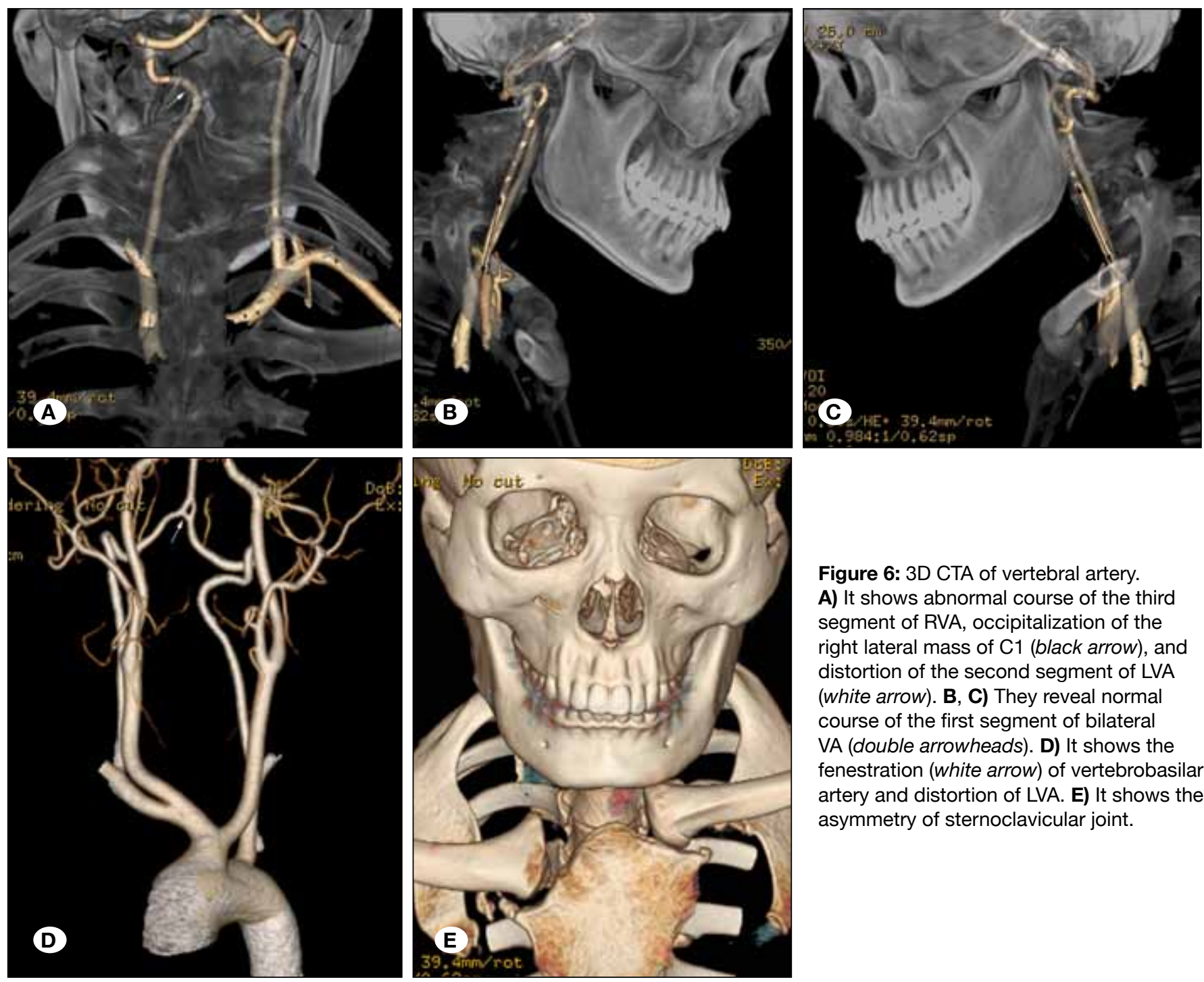

Figure 6: 3D CTA of vertebral artery. A) It shows abnormal course of the third segment of RVA, occipitalization of the right lateral mass of $\mathrm{C} 1$ (black arrow), and distortion of the second segment of LVA (white arrow). B, C) They reveal normal course of the first segment of bilateral VA (double arrowheads). D) It shows the fenestration (white arrow) of vertebrobasilar artery and distortion of LVA. E) It shows the asymmetry of sternoclavicular joint.
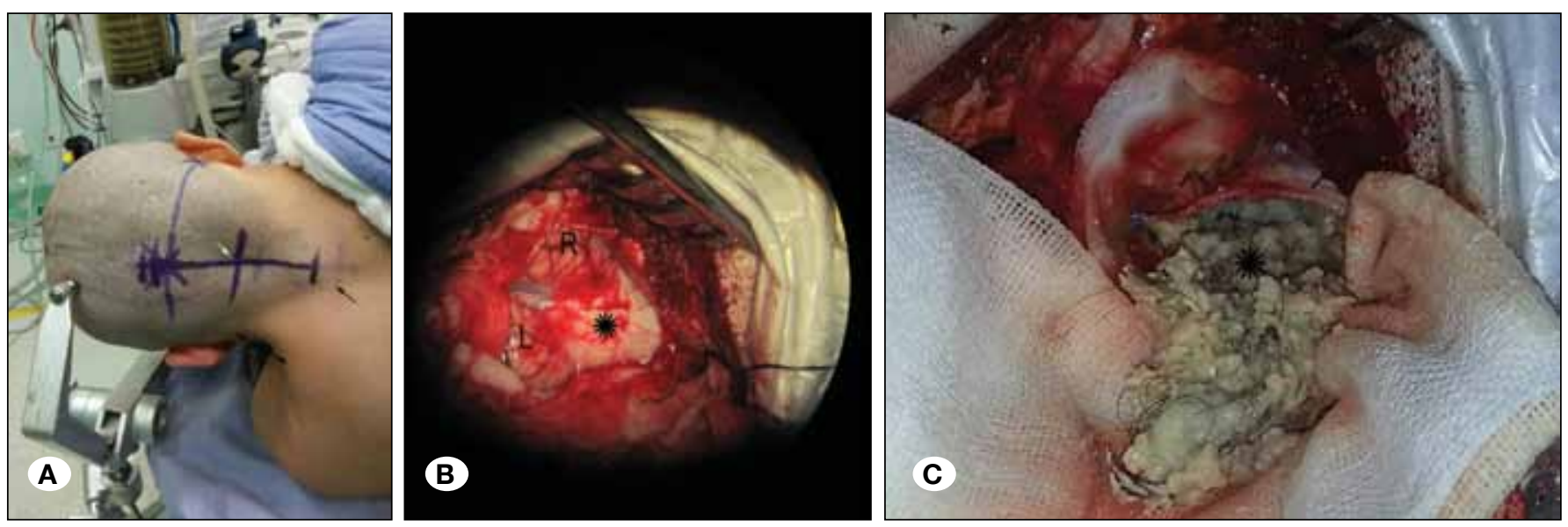

Figure 7: Intraoperative images of the patient. A) It shows the low occipital headline (black arrow), short neck (double arrowheads) and one apparent pit (white arrow). B) It reveals bulgy cerebellar tissue $(R, L)$ and tumor (asterisk). C) the contents in the cyst (asterisk). $(R, L$ shorting for right, left cerebellar hemisphere). 


\section{DISCUSSION}

Analysis of the 25 cases in Table II present more details concerning the association of KFS with DCs. These cases included 14 women and 11 men, with an average age of 19.7

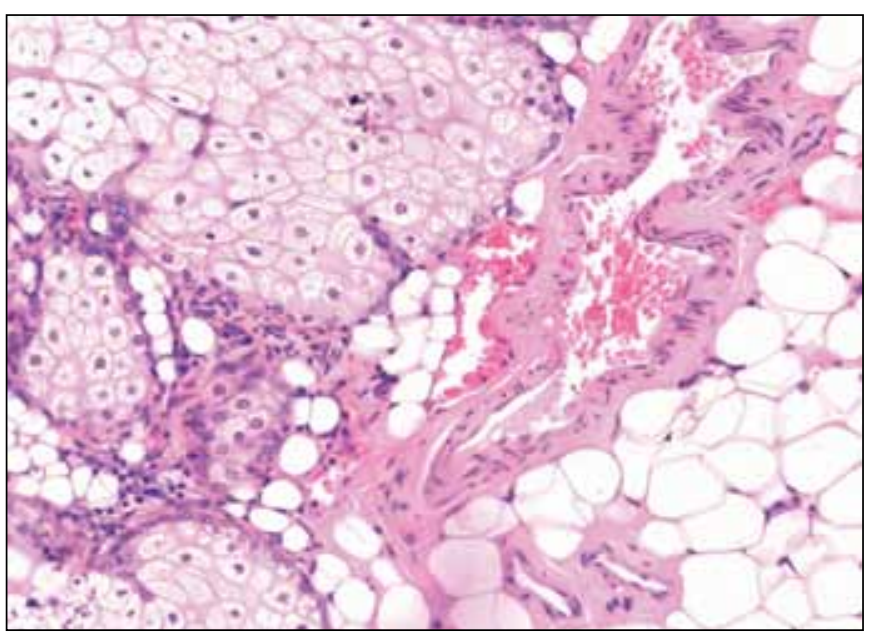

Figure 8: Photomicrograph demonstrating a classic DC with a lining of smooth muscle cells, sebaceous gland and keratinous material with calcification $(H \& E, \times 200)$. years (ranging from 1 to 61 years). Pediatric cases represented $56 \%$ of patients, indicating that approximately half of such patients are not diagnosed until adulthood. The follow-up period ranged from 3 months to 6 years among the 17 cases with sufficient information. Tumor recurrence was not reported in these patients during the follow-up period.

\section{Klippel-Feil Syndrome}

KFS is associated with the classic triad of a short neck, a low posterior hairline and a limited range of motion of the neck; it is caused by congenital fusion of two or more cervical vertebrae $(11,26)$. Analysis of the 25 cases shows that the classic KFS triad is present in $37.5 \%$ of patients. The lack of population screening studies has made it impossible to determine the exact incidence and prevalence of KFS (39). KFS can be classified into three subtypes (11) - type I, II or III:

Type I is defined as having multiple cervical or upper thoracic vertebral fusions;

Type II is defined as having isolated fusions at 1 or 2 cervical interspaces;

Type III is defined as having fusions in the cervical spine combined with lower thoracic or lumbar fusion.

Therefore, the current case was classified as Type I of KFS. As shown in Tables I and II, vertebral fusions were described in 21

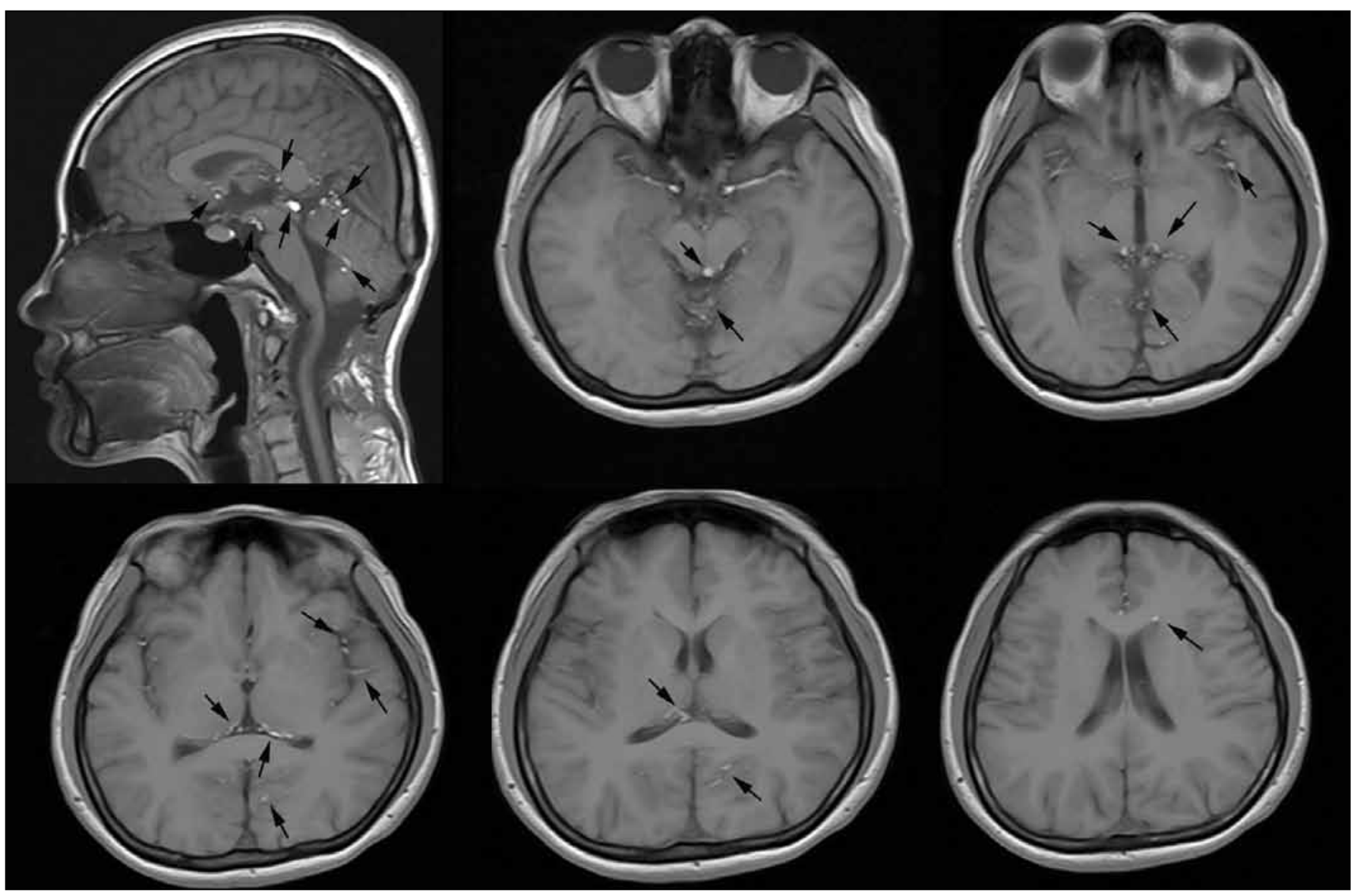

Figure 9: T1 MRI follow-up scans show the fat droplets (arrows) in the brain. 
cases, with $62 \%$ classified as type I, $33.3 \%$ classified as type II, and $4.7 \%$ classified as type III. Furthermore, it is unclear whether the diagnosis of KFS is a discrete entity or if it is one condition in a spectrum of congenital spinal deformities (12). As described in Table I, congenital deformities associated with KFS and DCs include Sprengel's deformity, asymmetry of the sternoclavicular joint, fusion of the ribs or rudimentary cervical ribs, pigeon chest, palatal fissure, and basilar artery fenestration et al.

In recent years, numerous studies of KFS have been reported, particularly regarding the genetic etiology, such as a paracentric inversion on $8 q(6)$, mutations of MEOX1 gene $(2,24)$, Notch signaling pathway $(33,36)$, Pax 1 and Pax 9 (5), or HOX gene (21). However, most of these conditions have only been confirmed in animal studies, and further studies are warranted to investigate the exact genetic origins of KFS.

\section{Dermoid Cysts}

The common view is that there is a focal failure of cleavage between the neuroectoderm and cutaneous ectoderm between the third and fifth weeks of embryogenesis (15, 41). This results in the incorporation of cutaneous ectoderm, which forms the skin epithelium and all skin appendages, into the neural tube. Additionally, dermoid or epidermoid cysts of the CNS may be produced $(4,10,34,37)$. The clinical manifestations of DCs are non-specific and insidious, unless rupture occurs (14). Symptoms in patients with unruptured DCs result from compression of adjacent neurovascular structures. As shown in tables 1 and 2, DC patients were often admitted with signs $(41.7 \%)$ of elevated intracranial pressure (rICP), in addition to cerebellar signs (33.3\%). Rarely, ruptured intracranial DCs could result in dissemination of fatty material into cisterns, producing variable neurological symptoms, such as chemical meningitis (43), seizures (18), visual deficits (38), hydrocephalus (14), transient cerebral ischemia (45), or no symptoms, just as in our case. Additionally, rupture is usually spontaneous and is due to an increase in the size of the tumor's contents, together with head movements and/or brain pulsations; however, rupture has been reported following head trauma $(16,30)$. According to Table II, DCs located in the midline of the posterior fossa were found in 21 cases (84\%), DCs extending to the craniocervical junction (including the present case) were found in 3 cases (12\%), and a DC in the cerebellar hemisphere was found in one case (4\%), showing that the midline of the posterior fossa is the most common site of DCs. Complete resection of DCs was achieved in $86 \%$ of cases. Additionally, $87.5 \%$ (21/24) of patients did not require OC fusion and did not demonstrate instability; instead, there was one patient with mild restriction of neck movement after OC fusion.

\section{Craniovertebral Junction Anomalies}

The bony CVJ can be divided into two components: a central pillar, which consists of the clivus and a central pivot (including the dens and $\mathrm{C} 2$ vertebral body), and a bistratal ring around the central pivot. They are the foramen magnum ring superiorly and the atlantal ring inferiorly (29). Therefore, developmental anomalies at the CVJ can also be classified into those related to the two components. As shown in Tables I and II, atlas assimilation is the most common CVJ anomaly, followed by platybasia, basilar invagination, atlantoaxial dislocation (AAD) and spina bifida occulta. A defect in the posterior arch of $\mathrm{C} 1$ also occurred in the present patient.

\section{Mirror Movement}

Mirror movement, also known as synkinesia or Kallmann's syndrome (22), occurs when voluntary movements of one side of the body are copied involuntarily by the other side of the body. This synkinesia manifests as a rare disorder that is usually associated with KFS $(31,35)$. It may represent neurological impairment or agenesis of the pyramidal decussation (44), cerebral cortex, corpus callosum, medulla oblongata or spinal cord (31).

To date, however, according to Table II, only three cases of KFS and posterior fossa DC have been reported with mirror movement. In our case, we believe that neurological impairment in the pyramidal decussation and the medulla oblongata, due to long-term compression by a congenital tumor, may be the cause of mirror movement.

\section{CONCLUSION}

This is the first reported case of associated CVJ anomalies, KFS, ruptured DC and mirror movement in one patient. A literature review discusses these conditions from multiple aspects. Several hypotheses $(4,15)$ have been proposed to explain the association among posterior fossa DC, KFS, and CVJ anomalies. Additionally, the combination of $\mathrm{CVJ}$ anomalies, KFS and DC may represent a new syndrome that has previously gone unnoticed. 


\begin{tabular}{|c|c|c|c|c|c|c|c|c|c|c|}
\hline $\begin{array}{l}\frac{1}{3} \\
\frac{0}{\overline{0}} \\
\text { 을 }\end{array}$ & 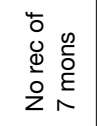 & : & 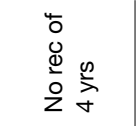 & i & 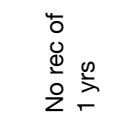 & : & : & : & 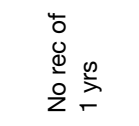 & 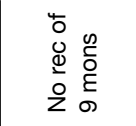 \\
\hline 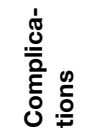 & zo & : & z & : & z & z & z & : & & z \\
\hline 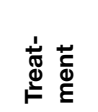 & 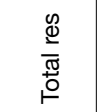 & 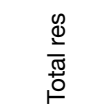 & 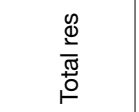 & 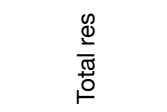 & 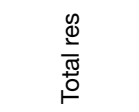 & 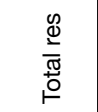 & 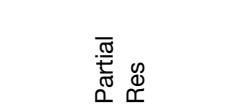 & i & 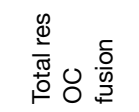 & 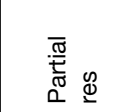 \\
\hline 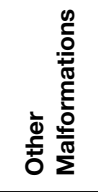 & $\stackrel{0}{z}$ & i & $i$ & i & : & i & 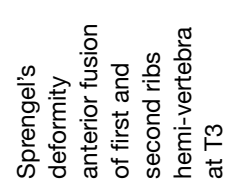 & i & 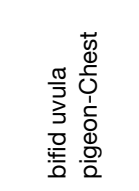 & : \\
\hline 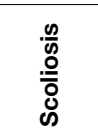 & zo & : & : & : & : & : & 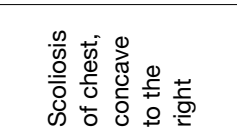 & : & : & 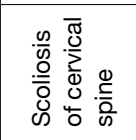 \\
\hline 总 & 1 & i & i & i & 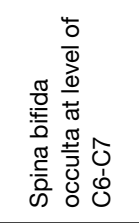 & 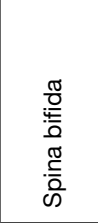 & 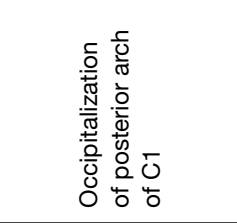 & i & 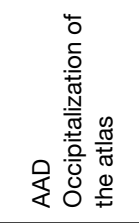 & 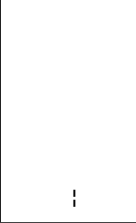 \\
\hline $\begin{array}{l}\frac{.0}{0} \\
\frac{9}{4} \\
\end{array}$ & $\begin{array}{l}\text { ஸे } \\
\text { ஸ் }\end{array}$ & 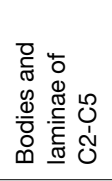 & 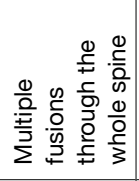 & 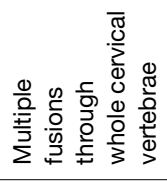 & 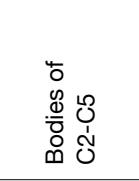 & $\begin{array}{l}\text { ญे } \\
\text { ஸे }\end{array}$ & 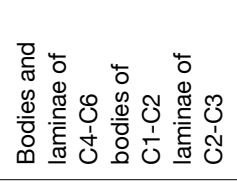 & $i$ & 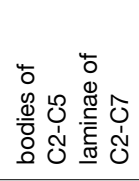 & i \\
\hline 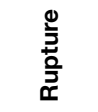 & \& & \& & \& & 운 & z & : & z & 을 & 우 & 운 \\
\hline 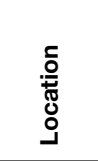 & 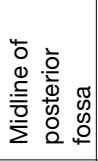 & 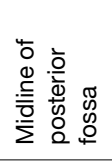 & 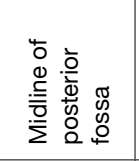 & 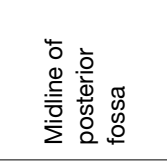 & 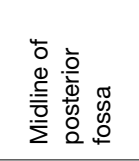 & गे & 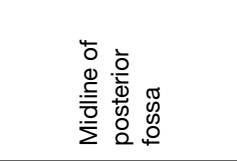 & 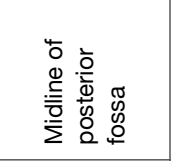 & 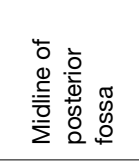 & 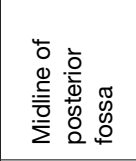 \\
\hline 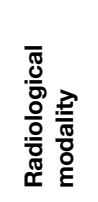 & 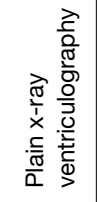 & 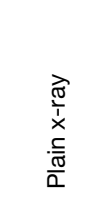 & 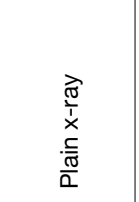 & 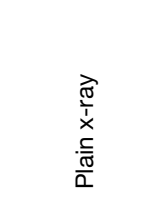 & 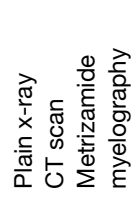 & 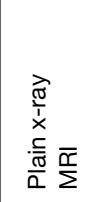 & 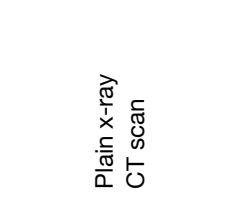 & 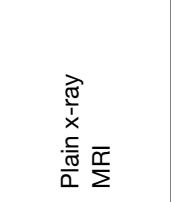 & 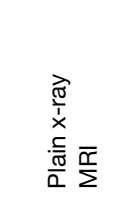 & $\overline{\frac{\alpha}{\Sigma}}$ \\
\hline 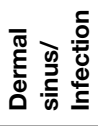 & $\begin{array}{l}\stackrel{0}{z} \\
\stackrel{0}{z}\end{array}$ & 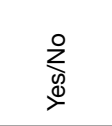 & 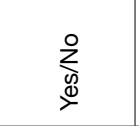 & 旡 & 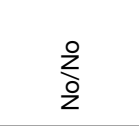 & 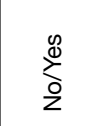 & 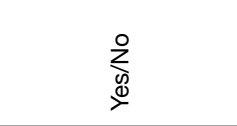 & $i$ & $\begin{array}{l}\stackrel{0}{z} \\
\text { ò }\end{array}$ & 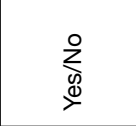 \\
\hline 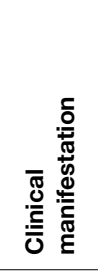 & 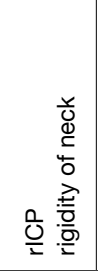 & 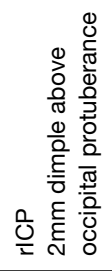 & $\frac{0}{\frac{0}{2}}$ & 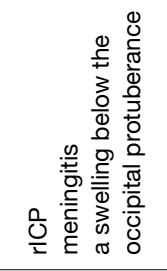 & 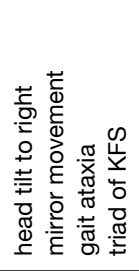 & 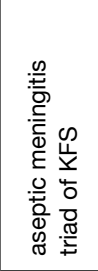 & 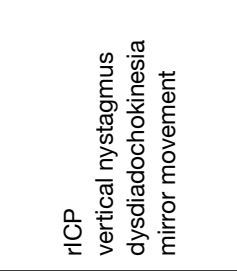 & 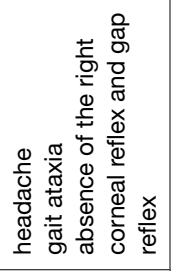 & 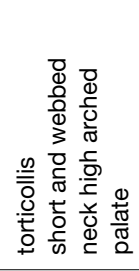 & 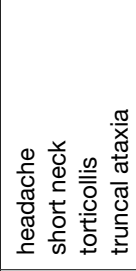 \\
\hline \begin{tabular}{l}
$\bar{D}$ \\
\hdashline
\end{tabular} & $\begin{array}{l}\text { u. } \\
\text { o }\end{array}$ & $\frac{4}{6}$ & $\frac{4}{60}$ & 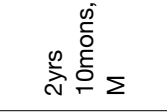 & $\stackrel{u}{s}$ & $\begin{array}{l}u \\
\stackrel{u}{y}\end{array}$ & $\begin{array}{l}\stackrel{u}{+} \\
\dot{J}\end{array}$ & $\begin{array}{l}u \\
\text { ó }\end{array}$ & $\begin{array}{l}u \\
\stackrel{u}{\sim}\end{array}$ & $\sum_{\infty}^{\infty}$ \\
\hline 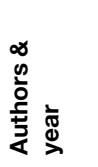 & 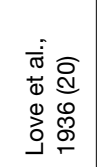 & 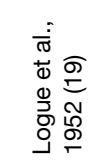 & 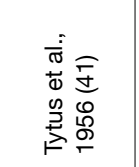 & 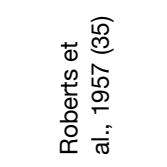 & 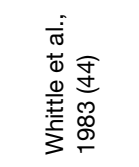 & 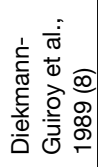 & 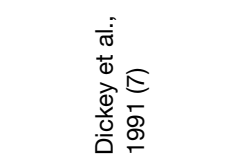 & 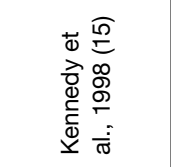 & 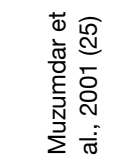 & \\
\hline
\end{tabular}


Zhang YK. et al: CVJ, KFS, DC, Mirror Movement

\begin{tabular}{|c|c|c|c|c|c|c|c|c|}
\hline 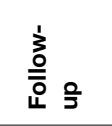 & 1 & 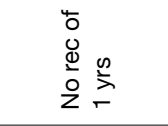 & : & 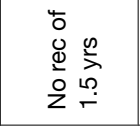 & 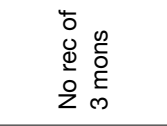 & 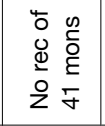 & 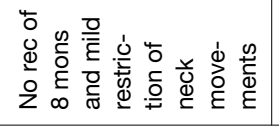 & 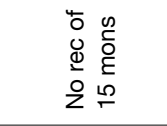 \\
\hline 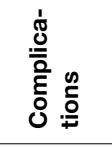 & $i$ & : & z & z & o & 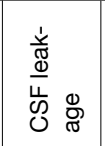 & 2 & 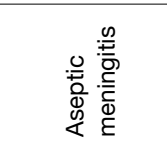 \\
\hline 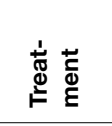 & : & 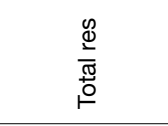 & 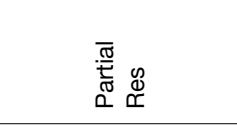 & 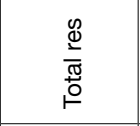 & 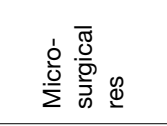 & 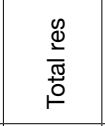 & 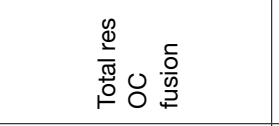 & $\mathscr{g}$ \\
\hline 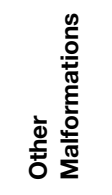 & 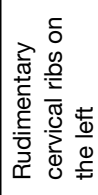 & : & 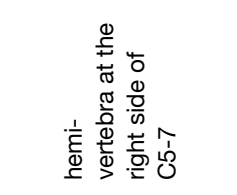 & 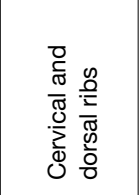 & : & : & : & $i$ \\
\hline 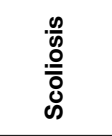 & $i$ & i & 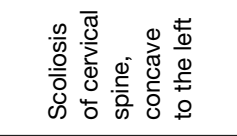 & i & i & ! & : & i \\
\hline 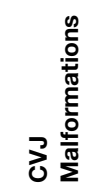 & 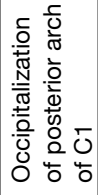 & i & 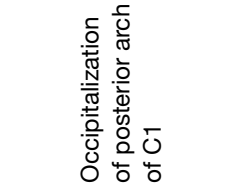 & 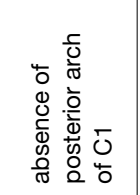 & 2 & : & 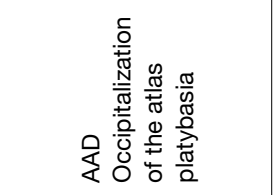 & 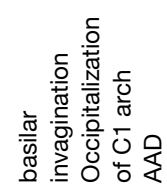 \\
\hline$\frac{5}{\frac{0}{0}}$ & 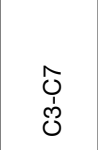 & 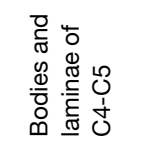 & $\begin{array}{l}\text { Ò్ } \\
\text { ป் }\end{array}$ & 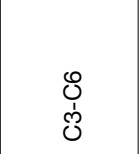 & $\begin{array}{l}\mathscr{1} \\
\dot{\delta}\end{array}$ & : & $\begin{array}{l}\text { O̦ } \\
\text { ஸ் }\end{array}$ & $\begin{array}{l}\text { O̦ } \\
\text { ஸ் }\end{array}$ \\
\hline 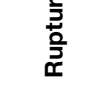 & z & z & z & z & ㅇ & ㅇ & z & $\stackrel{\circ}{z}$ \\
\hline 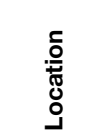 & 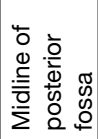 & 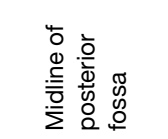 & 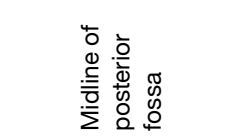 & 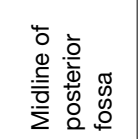 & 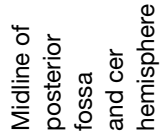 & 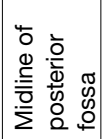 & 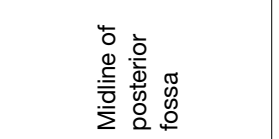 & 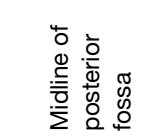 \\
\hline 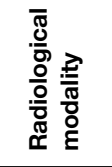 & 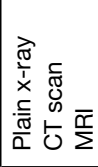 & 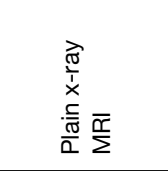 & 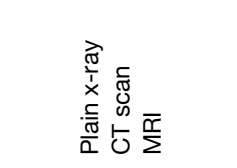 & 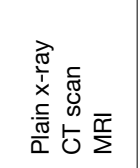 & $\overline{\frac{\tilde{x}}{\Sigma}}$ & $\stackrel{\overleftarrow{v}}{\Sigma}$ & 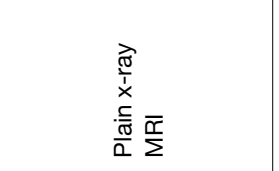 & 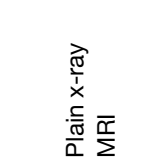 \\
\hline 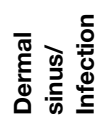 & 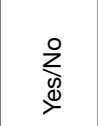 & 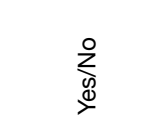 & $i$ & 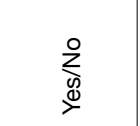 & $\frac{1}{2}$ & 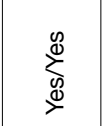 & $\begin{array}{l}\stackrel{0}{z} \\
\text { o }\end{array}$ & $\begin{array}{l}\stackrel{0}{z} \\
\text { o }\end{array}$ \\
\hline 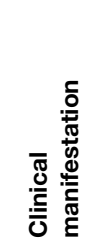 & 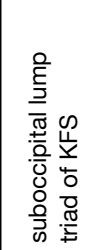 & 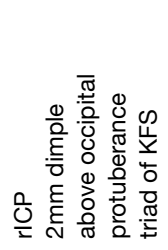 & 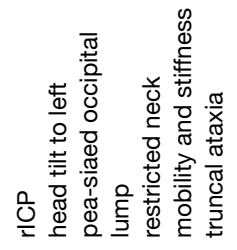 & 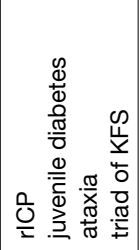 & : & 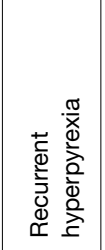 & 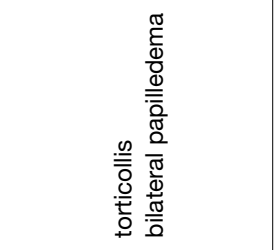 & 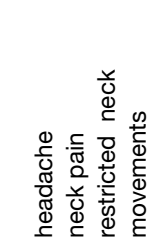 \\
\hline 离希 & $\begin{array}{l}\sum_{0} \\
0 \\
\underline{0}\end{array}$ & $\stackrel{u}{\stackrel{u}{N}}$ & $\sum$ & $\begin{array}{l}\sum_{0} \\
i\end{array}$ & $\begin{array}{l}\stackrel{4}{5} \\
\text { if }\end{array}$ & $\sum_{i}$ & $\sum_{\substack{\text { N } \\
\text { N }}}$ & 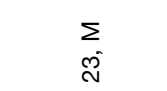 \\
\hline 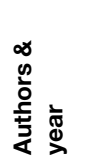 & 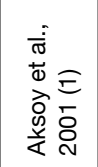 & 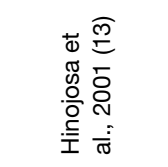 & 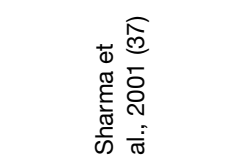 & 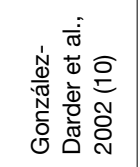 & 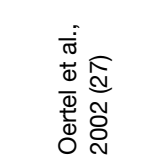 & 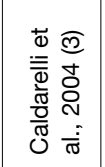 & 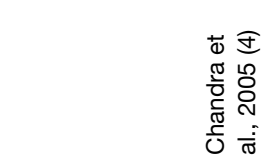 & \\
\hline
\end{tabular}




\begin{tabular}{|c|c|c|c|c|c|c|c|c|}
\hline 咅 & 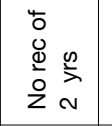 & i & $\begin{array}{l}\frac{0}{0} \\
\frac{\Phi}{2} \\
z\end{array}$ & 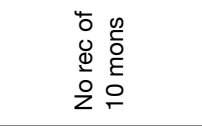 & 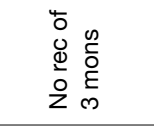 & 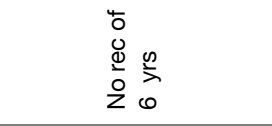 & 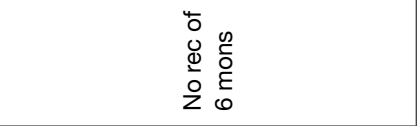 & 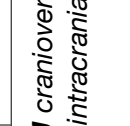 \\
\hline 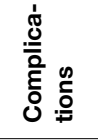 & 1 & 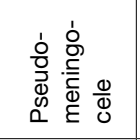 & 2 & 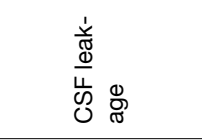 & \& & 2 & 2 & 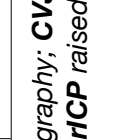 \\
\hline 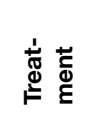 & $\stackrel{\mathscr{g}}{\underline{\underline{D}}}$ & $\stackrel{\mathscr{\infty}}{\underline{\infty}}$ & 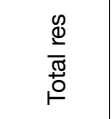 & 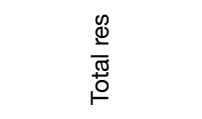 & 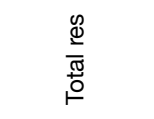 & 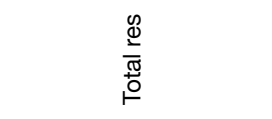 & 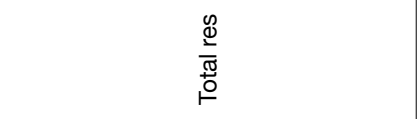 & 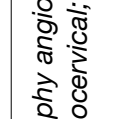 \\
\hline 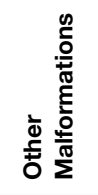 & 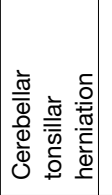 & 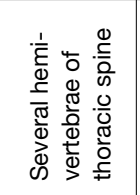 & : & 을 & z & 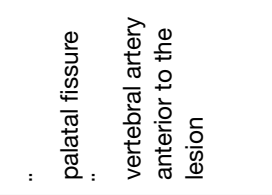 & 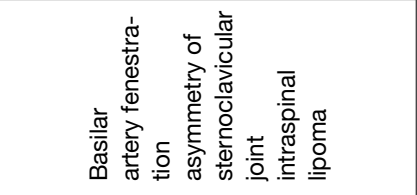 & 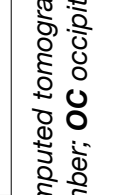 \\
\hline $\begin{array}{l}\frac{\infty}{0} \\
\stackrel{0}{0} \\
\dot{D} \\
\infty\end{array}$ & : & : & i & 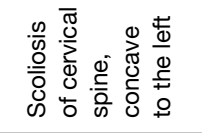 & : & 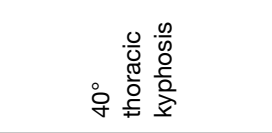 & 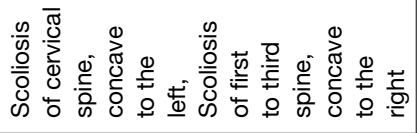 & 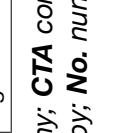 \\
\hline 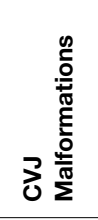 & $i$ & $i$ & 1 & $\stackrel{\circ}{z}$ & $\stackrel{0}{z}$ & 으 & 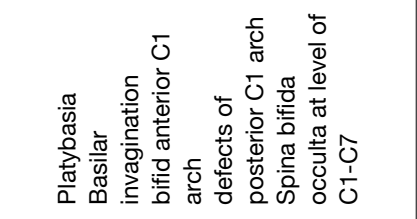 & 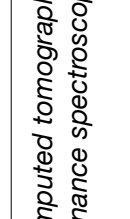 \\
\hline $\begin{array}{c}\frac{0}{0} \\
\frac{0}{3} \\
\frac{1}{4}\end{array}$ & \begin{tabular}{l}
\multirow{0}{0}{} \\
$\dot{U}$ \\
Oे
\end{tabular} & 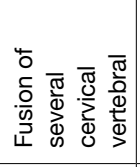 & i & $\begin{array}{l}\hat{O} \\
\dot{y} \\
0\end{array}$ & $\begin{array}{l}0 \\
\dot{\delta} \\
\tilde{\delta}\end{array}$ & 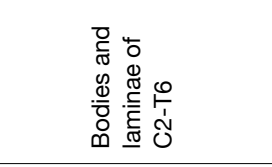 & 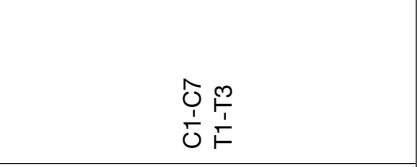 & 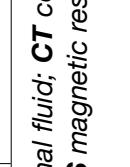 \\
\hline 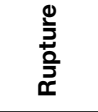 & z & $\stackrel{\circ}{z}$ & 2 & 2 & 우 & 우 & $\stackrel{\infty}{\nu}$ & 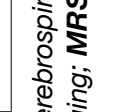 \\
\hline 产 & 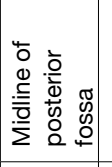 & 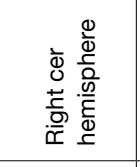 & 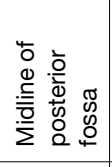 & 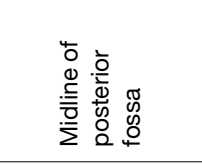 & 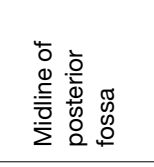 & 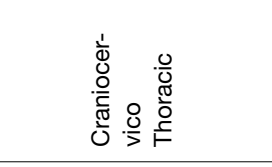 & 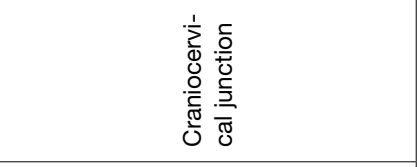 & 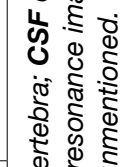 \\
\hline 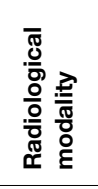 & 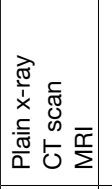 & 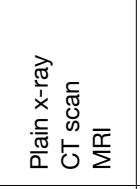 & 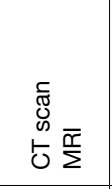 & 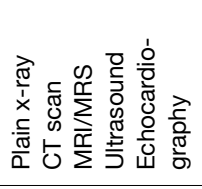 & 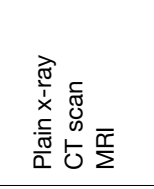 & 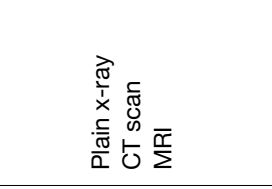 & 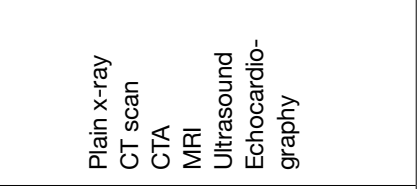 & 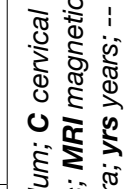 \\
\hline 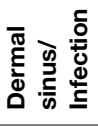 & $\begin{array}{l}\stackrel{0}{z} \\
\text { i }\end{array}$ & $\begin{array}{l}\frac{1}{D} \\
\stackrel{\infty}{x}\end{array}$ & 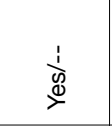 & $\sum_{\substack{\infty \\
ٍ}}^{0}$ & $\underset{\substack{0 \\
\nu}}{\stackrel{0}{\nu}}$ & $\sum_{\substack{\infty\\
}}^{0}$ & $\begin{array}{l}\stackrel{0}{z} \\
\text { o }\end{array}$ & 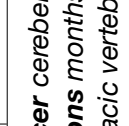 \\
\hline 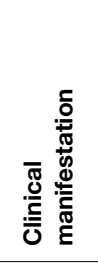 & 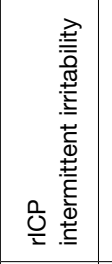 & 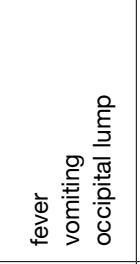 & 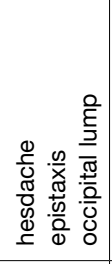 & 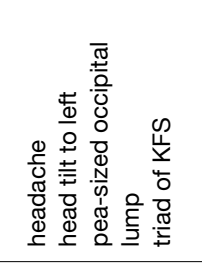 & 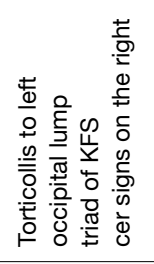 & 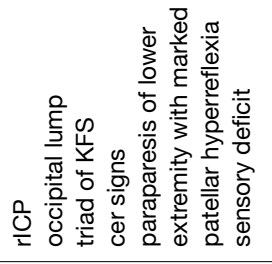 & 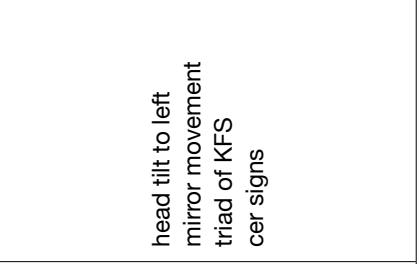 & 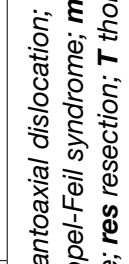 \\
\hline 高 & $\sum_{\infty}$ & $\sum$ & $\sum_{N}$ & $\begin{array}{l}L \\
\dot{\sigma}\end{array}$ & $\begin{array}{l}\text { Lu } \\
\omega^{\circ}\end{array}$ & \begin{tabular}{l}
$\stackrel{L}{*}$ \\
\multirow{v}{*}{}
\end{tabular} & 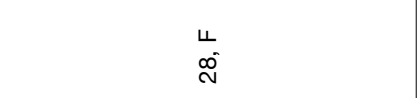 & 东趁 \\
\hline 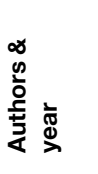 & & 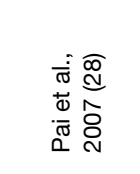 & & 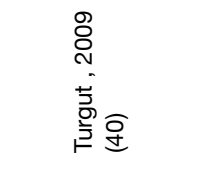 & 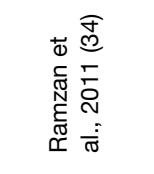 & 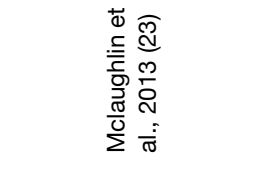 & 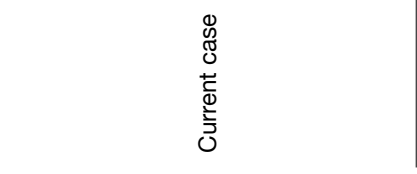 & 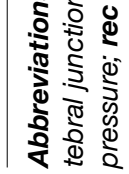 \\
\hline
\end{tabular}


Table II: Further Analysis of the 25 Reported Cases (Including the Present Case)

Details

No. (\%)

No. Cases

Details

No. $(\%)$

where data is specified

No. of Patients and Avg Age [range]yrs

Female [Avg age] yrs

Male [Avg age] yrs

Patients $\leq 18 y r s$ [Avg age] yrs
25 and 19.7 [1-61]
14 [26.3]
$11[11.4]$
14 [6] $(56 \%)$

25

\section{Clinical manifestation \\ Dermal sinus \\ Occipital dimple or lump \\ rICP \\ Triad of KFS \\ Cerebellar signs \\ DC location \\ Midline of posterior fossa \\ CVJ \\ Cerebellar hemisphere}

Short neck or restriction of neck or head titling to one side meningitis
$14(58.3 \%)$
$10(41.7 \%)$
$10(41.7 \%)$
$9(37.5 \%)$
8 (33.3\%)
7 (29.2\%)
$1(4.2 \%)$

21 (84\%)

$3(12 \%)$

25

$1(4 \%)$

\section{Types of KFS}

$\begin{array}{ll}\text { I } & (62 \%) \\ \text { II } & (33.3 \%) \\ \text { III } & (4.7 \%) \\ \text { unmentioned } & 4\end{array}$
(33.3\%)
$(4.7 \%)$

21

\section{CVJ malformations}

Atlas assimilation

Basiocciput dysplasia (platybasia, basilar invagination)

Dysplasia of the lateral sclerotome or/and hypochordal bow (hypoplasia or

Defects of anterior or/and posterior C1 arch)

AAD

Spina bifida occulta

$$
\begin{aligned}
& 6(60 \%) \\
& 3(30 \%) \\
& 2(20 \%) \\
& 3(30 \%) \\
& 3(30 \%)
\end{aligned}
$$

\begin{tabular}{l}
\hline Ruptured DC \\
\hline Mirror movement \\
\hline Surgical resection \\
Resection and OC fusion \\
Detailed described resection \\
Total resection \\
Partial resection
\end{tabular}

\section{Complication of post-operation}

CSF leakage

Aseptic meningitis

Pseudo-meningocele

\section{Follow-up}

Avg time [range] months

Tumor recurrence

Mild restriction of neck movement of $\mathrm{OC}$ fusion

$\begin{array}{cc}3(12.5 \%) & 24 \\ 21 & 24 \\ 18(86 \%) & 21 \\ 3(14 \%) & 21\end{array}$

(8.3\%)

24

$1(4.1 \%)$

24

$1(4.1 \%)$

24

Abbreviations: AAD atlantoaxial dislocation; Avg average; CSF cerebrospinal fluid; CVJ craniovertebral junction; DC dermoid cyst; No. number; OC Occipitocervical; rICP raised intracranial pressure. 


\section{- REFERENCES}

1. Aksoy FG, Aksoy OG, Gomori JM: Klippel-Feil syndrome in association with posterior fossa suboccipital dermoid cyst. Eur Radiol 11:142-144, 2001

2. Bayrakli F, Guclu B, Yakicier C, Balaban H, Kartal U, Erguner B, Sagiroglu MS, Yuksel S, Ozturk AR, Kazanci B, Ozum U, Kars $\mathrm{HZ}$ : Mutation in MEOX1 gene causes a recessive Klippel-Feil syndrome subtype. BMC Genetics 14: 95, 2013

3. Caldarelli M, Massimi L, Kondageski C, Rocco CD: Intracranial midline dermoid and epidermoid cysts in children. J Neurosurg Pediatr 100: 473-480, 2004

4. Chandra PS, Gupta A, Mishra NK, Mehta VS: Association of craniovertebral and upper cervical anomalies with dermoid and epidermoid cysts: Report of four cases. Neurosurgery 56:1155, 2005

5. Chi N, Epstein JA: Getting your Pax straight: Pax proteins in development and disease. Trends Genet 18: 41-47, 2002

6. Clarke RA, Singh S, Mckenzie H, Kearsley JH, Yip MY: Familial Klippel-Feil syndrome and Paracentric inversion inv(8) (q22.2q23.3). Am J Hum Genet 57: 1364-1370, 1995

7. Dickey W, Hawkins SA, Kirkpatrick DH, Gray WJ: Posterior fossa dermoid cysts and the Klippel-Feil syndrome. J Neurol Neurosurg Psychiatry 54: 1016-1017, 1991

8. Diekmann-Guiroy B, Huang PS: Klippel-Feil syndrome in association with a Craniocervical dermoid cyst presenting as aseptic meningitis in an adult: Case report. Neurosurgery 25:652655, 1989

9. Gallea G, Popa T, Hubsch C, Valabregue R, Brochard V, Kundu P, Schmitt B, Bardinet E, Bertasi E, Flamand-Roze C, Alexandre N, Delmaire C, Méneret A, Depienne C, Poupon C, Hertz-Pannier L, Cincotta M, Vidailhet M, Lehericy S, Meunier S, Roze E: RAD51 deficiency disrupts the corticospinal lateralization of motor control. Brain 136: 3333-3346, 2013

10. González-Darder JM, Feliu-Tatay R, Pesudo-Martínez JV, VeraRomán JM: Klippel-Feil syndrome associated with posterior fossa dermoid cyst. Case report. Neurol Res 24:501-504, 2002

11. Gunderson CH, Greenspan RH, Glaser GH, Lubs HA: The KlippelFeil syndrome: Genetic and clinical reevaluation of cervical fusion. Medicine 46: 491-512, 1967

12. Hensinger RN, Lang JE, Macewen GD: Klippel-Feil syndrome: $A$ constellation of associated anomalies. J Bone Joint Surg 56-A: 1246-1253, 1974

13. Hinojosa M, Tatagiba M, Harada K, Samii M: Dermoid cyst in the posterior fossa accompanied by Klippel-Feil syndrome. Childs Nerv Syst 17:97-100, 2001

14. Karabulut N, Oguzkurt L: Tetraventricular hydrocephalus due to ruptured intracranial dermoid cyst. Eur Radiol 10: 1810-1811, 2000

15. Kennedy PT, McAuley DJ: Association of posterior fossa dermoid cyst and Klippel-Feil syndrome. AJNR Am J Neuroradiol 19:195196, 1998

16. Kim IN, Jung S, Jung TY, Kang SS, Kim TS: Traumatic rupture of an intracranial dermoid cyst. J Clin Neurosci 15: 469-471, 2008

17. Kim KH, Cho JH: Ruptured intracranial dermoid cyst associated with rupture of cerebral aneurysm. J Korean Neurosurg Soc 50: 453-456, 2011

18. Kucera JN, Roy P, Murtagh R: Ruptured intracranial dermoid cyst manifesting as new onset seizure: A case report. Neuroradiology 5: $10-18,2011$
19. Logue V, Till K: Posterior fossa dermoid cysts with special reference to intracranial infection. J Neurol Neurosurg Psychiatry 15: 1-12, 1952

20. Love JG, Kernohan JW: Dermoid and epidermoid tumors (cholesteatomas) of central nervous system. JAMA 107: 18761883, 1936

21. Manzanares $M$, Wada $H$, Itasaki N, Trainor PA, Krumlauf R, Holland PW: Conservation and elaboration of Hox gene regulation during evolution of the vertebrate head. Nature 408: 854-857, 2000

22. Mayston MJ, Harrison LM, Quinton R, Stephens JA, Krams M, Bouloux PM: Mirror movement in X-linked Kallmann's syndrome. I. A neurophysiological study. Brain 12: 1199-1216, 1997

23. Mclaughlin N, Weil AG, Demers J, Shedid D: Klippel-Feil syndrome associated with a craniocervico-thoracic dermoid cyst. Surg Neurol Int 4:S61-66, 2013

24. Mohamed JY, Faqeih E, Alsiddiky A, Alshammari MJ, Ibrahim NA, Alkuraya FS: Mutations in MEOX1, Encoding Mesenchyme Homebox 1, cause Klippel-Feil anomaly. Am J Hum Genet 92: $157-161,2013$

25. Muzumdar D, Goel A: Posterior cranial fossa dermoid in association with Craniovertebral and cervical spinal anomaly: Report of two cases. Pediatr Neurosurg 35:158-161, 2001

26. Nagib MG, Maxwell RE, Chou SN: Klippel-Feil syndrome in children: Clinical features and management. Childs Nerv Syst 1: 255-263, 1985

27. Oertel J, Piek J, Müller JU, Vogelgesang S, Warzok R, Gaab RM: Posterior fossa squamous cell carcinoma due to dedifferentiation of a dermoid cyst in Klippel-Feil syndrome case illustration. J Neurosurg 97:1244, 2002

28. Pai VV, Lowe LH, Castillo M, Huang BY, Shao L: Posterior fossa dermoid cysts in association with Klippel-Feil syndrome: Report of three cases. AJNR Am J Neuroradiol 28:1926-1928, 2008

29. Pang D, Thompson D: Embryology and bony malformations of the craniovertebral junction. Childs Nerv Syst 27:523-564, 2011

30. Park SK, Cho KG: Recurrent intracranial dermoid cyst after subtotal removal of traumatic rupture. Clin Neurol Neurosurg 114: 421-424, 2012

31. Parmar PN, Seta AA, Solanki AB: Congenital mirror movements in nodular heterotopia and partial block vertebrae. J Pediatr Neurol 1: 43-49, 2013

32. Pinninti R, Thirulogachandar E, Noorul Ameen KH: An interesting clinical association of short neck with an unusual laryngeal anomaly. J Craniovert Jun Spine 2: 86-88, 2011

33. Pourquié $\mathrm{O}$, Kusumi $\mathrm{K}$ : When body segmentation goes wrong. Clin Genet 60: 409-416, 2001

34. Ramzan A, Khursheed N, Rumana M, Abrar W, Ashish J: Posterior fossa dermoid with Klippel-Feil syndrome in a child. Pediatr Neurol 45:197-199, 2011

35. Roberts AP: A case of intracranial dermoid cyst associated with the Klippel-Feil deformity and recurrent meningitis. Arch Dis Child 33:222-225, 1958

36. Saga $\mathrm{Y}$, Takeda $\mathrm{H}$ : The making of the somite: Molecular events in vertebrate segmentation. Nat Rev Genet 2: 835-845, 2001

37. Sharma MS, Sharma BS, Yadav A, Khosla VK: Posterior fossa dermoid in association with Klippel-Feil syndrome--a short report. Neurol India 49:210-212, 2001

38. Skovrlj B, Mascitelli JR, Steinberger JM, Weiss N: Progressive visual loss following rupture of an intracranial dermoid cyst. J Clin Neurosci 21: 159-161, 2014 
39. Tracy MR, Dormans JP, Kusumi K: Klippel-Feil syndrome: Clinical features and current understanding of etiology. Clin Orthop Relat Res 424:183-190, 2004

40. Turgut M: Klippel-Feil syndrome in association with posterior fossa dermoid tumor. Acta Neurochir 151: 269-279, 2009

41. Tytus JS, Pennybacker J: Pearly tumours in relation to the central nervous system. J Neurol Neurosurg Psychiatry 19: 241-259, 1956

42. Wang SL, Wang C, Liu Y, Yan M, Zhou HT: Anomalous vertebral artery in craniovertebral junction with occipitalization of the atlas. Spine 34:2838-2842, 2009
43. Wang YM, Chang TP, Lo CP, Tu MC: Spontaneous rupture of intracranial dermoid cyst with chemical meningitis. J Emerg Med 44: e275-e276, 2013

44. Whittle IR, Besser M: Congenital neural abnormalities presenting with mirror movements in a patient with Klippel-Feil syndrome. J Neurosurg 59: 891-894, 1983

45. Zheng K, Mao BY, Ma L, Jiang S: Ruptured intracranial dermoid cyst with infarction in the basal ganglia: Case report. Neurol Med Chir (Tokyo) 50: 254-256, 2010 